\title{
ON PROPERTIES OF A DOMAIN FOR WHICH ANY DERIVED SET IS CLOSED*
}

B)

\section{EARLE RAYMOND HEDRICK}

1. Introduction. Frechet, in his thesis $\dagger$, defines a general class of assemblages $(L)$ of objects of any sort, with which some definition of limiting element of a sequence is associated. $f$

The most interesting type of such assemblages is that which Frecher calls compact. $\S$ Among such assemblages one very important class are those which have the property that the first derived set $\left(E^{\prime}\right)$ of every subset $(E)$ of $(D)$ is closed.\|

In this paper, some of the properties of any fundamental assemblage $(D)$ which has both the properties described above will be discussed. We shall call $(D)$ the fundamental domain. $T$

2. Elements Interior to Assemblages. Let there be given a fundamental domain $(D)$ with the properties** described in $\S 1$. An element $A$ is said to be interior + to a set $(E)$ of $(D)$ if $A$ is not a limiting element of any sequence of elements of $(D)$ which do not belong to $(E)$. One set $(E)$ is interior to another set $\left(F^{\prime}\right)$ if every element of $\left(E^{\prime}\right)$ is interior to $\left(F^{\prime}\right)$.

* Presented to the Society (Columbia, Mo.) November 27, 1909.

† Rendiconti del Circolo Matematico di Palermo, vol. 22 (1906).

$\ddagger$ The only restrictions are (1) that a sequence of identical elements, $A, A, \cdots, A, \cdots$ be said to have $A$ as a limiting element, (2) that if a sequence $A_{1}, A_{2}, \cdots, A_{n}, \cdots$ has a unique limit, any infinite subsequence selected from it, in the same order, has the same limit. (FRÉCHET, loc. cit., § 7.)

8 A fundamental domain is compact if every infinite set of its elements has at least one limiting element. (FRÉCHET, loo. cit., § 9.)

|| Compare FrÉCH LT, loc. cit., $\$ 22$. It is evident that domains exist which do not have this property. FrÉCH ET cites the case of the domain of all continuous functions defined in a closed interval. See also Schornflies, Punktmannigfaltigkeiten, Bericht der Deutsohen Mathematiker-Vereinigung, Ergänzungsband II (1908), page 86, lines 1-6, and page 279. On page 282 it is proved that this property is not a consequence of the compactness of $(D)$.

IT The notation $(L)$ is used by FrécheT for any domain for which an acceptable definition of limit is given. In this paper we shall assume that $(D)$ is compact and has the closure property mentioned above. The investigation of the properties of sets having these two characteristics is suggested by Schoenfliks, loc. cit., p. 279.

** The following theorems involve this hypothesis, which is not repeated below.

†† See FRÉCHET, loc. cit., § 8.

Trans. Am. Math. Soc. 20 
An immediate consequence of this definition is the following theorem :

Theorem 1. If an element $A$ is interior to a set $(E)$, all but a finite number of elements of any sequence whose limiting element is $A$ are interior to $(E)$. For if not, let $A_{1}, A_{2}, \ldots, A_{p}, \ldots$ be the infinite set of elements not interior to $(E)$ selected from such a sequence. Since $A_{i}$ is not interior to $(E)$, it can be approached by a sequence of elements $A_{i}^{(1)}, A_{i}^{(2)}, A_{i}^{(3)}, \ldots, A_{i}^{(k)}, \ldots$ of (D) which do not belong to $(E)$. The derivative of the set of elements $A_{i}^{(k)}$ contains the elements $A_{i}$. Since this derivative is closed, $A$ is one of its elements; hence $A$ can be approached by a sequence of elements of the set $A_{i}^{(k)}$ none of which belong to $(E)$. This is contrary to the hypothesis that $A$ is interior to $(E)$, and the theorem is proved.

3. The Heine-Borel Theorem. The theorem just proved enables us to show that the Heine-Borel theorem * holds for a fundamental domain $(D)$ which has the properties of section 1 .

Theorem 2. Let $\left(E_{1}\right),\left(E_{2}\right), \ldots,\left(E_{n}\right), \ldots$ be a countable set of assemblages, such that every element of a certain closed assemblage $(K)$ is interior to at least one of the assemblages $\left(E_{i}\right)$; then every element of $(K)$ is interior to one of a finite number of the assemblages $\left(E_{i}\right)$.

For suppose the theorem untrue. Let $A_{1}$ be an element of $(K)$ not interior to $\left(E_{1}\right)$, and let $\left(E_{n_{1}}\right)$ be the first of the $\left(E_{i}\right)$ to which $A_{1}$ is interior. Let $A_{2}$ be an element not in $\left(E_{j}\right), j \leqq n_{1}$; and let $\left(E_{n_{2}}\right)$ be the first $(E)$ to which $A_{2}$ is interior. Continuing this process indefinitely, as we may in case the theorem is untrue, we obtain an infinite sequence of elements $A_{i}$, such that $\left(E_{n_{i}}\right)$ is the first $(E)$ to which $A_{i}$ is interior, and $n_{i}>n_{i-1}$. The sequence $A_{i}$ has a limiting element $A$, which is itself interior to some $(E)$, say $\left(E_{q}\right)$. We may suppose $A$ is the only limiting element of the $A_{i}$, for, if not, a partial sequence can be selected out of the $A_{i}$ which has $A$ as its only limiting element and for which the essential properties of the $A_{i}$ are preserved. Since $A$ is then the only lim iting element of the $A_{i}$, it follows from Theorem 1 that all but a finite number of the $A_{i}$ are interior to $\left(E_{q}\right)$. This shows that the original process is fallacious, since $n_{i}=q$ for all sufficiently large values of $i$. Hence the theorem as stated must be correct.

4. Examples: The Hilbert Space. As an instance of a fundamental domain to which the preceding theorems apply we may cite, of course, any limited region of an ordinary space of one dimension (or in fact of $n$ dimensions), with the usual definition of limiting point. In fact, any assemblage of points in $n$

* The theorem is restricted to the case in which sets $E_{i}$ form a countable assemblage; this was the original form of the BoREL statement, afterwards generalized, for real variables, by LEBESGUE and others. FRÉCHeT gives (loc. cit., $\$ \S 36,42)$ proofs for any domain of type $(V)$ (ibid., $\S 27$, et seq.), under restrictions on the definition of distance (voisinage) and of limiting element which include the conditions of this paper (ibid., §28). The more general form of the theorem occurs in this paper as part of Theorem 16. 
dimensions which is itself closed may be chosen as the fundamental domain $(D)$. Such a domain evidently has the property that the first derived assemblage of any subset $(E)$ is closed. A more characteristic example, in which the definition of distance has nothing to do with the definition of limiting object is the Hilbert space of an infinite number of dimensions; ${ }^{*}$ the objects used are the points $\left(x_{1}, x_{2}, \ldots, x_{n}, \ldots\right)$ with the restriction $\Sigma x_{1}^{2} \leqq 1$. The definition of distance between two points is $\left[\Sigma\left(x_{1}^{(1)}-x_{2}^{(2)}\right)^{2}\right]^{\frac{1}{2}}$; and the points $\left(x^{(1)}\right),\left(x^{(2)}\right)$, $\cdots,\left(x^{(n)}\right), \ldots$ approach the point $(x)$ as a limit if $\lim _{n=\infty} x_{i}^{(n)}=x_{i}$ for every value of $i . \dagger$ This space evidently possesses the properties of $\S 1$; yet the set of points $\left(x^{(n)}\right)$ which approach $(x)$ may all lie at unit distance from $(x)$, as in the example $x_{i}^{(n)}=0$ if $n \neq i, x_{n}^{(n)}=1 ; x_{i}=0$. Frechet has given a definition of distance (voisinage) for which the preceding definition of limit points makes the distance between $\left(x^{(n)}\right)$ and $(x)$ approach zero. $\ddagger$ The preceding work shows that it is not always necessary to set up a definition of distance for the Hilbert space; for other domains of objects to do so might be very difficult or even impossible.

5. Other Theorems. Another property of sets of real numbers which holds for a fundamental domain $(D)$ having the properties of section 1 is the following : $\S$

Theorem 3. The set of elements at which the oscillation of any limited function $f(x)$ is not less than a given number $k$ is closed. Let $S$ be any sequence of elements $A_{1}, A_{2}, A_{3}, \ldots, A_{i}, \ldots$ whose only limiting element is $A$; let $M_{1}(A, n)$ be the upper limit of the values of $f(x)$ at the points $A_{n+p}(p=1,2, \cdots)$; then $M_{s}(A)=\lim _{n=\infty} M_{s}(A, n)$ surely exists and is called the maximum of $f(x)$ at $A$ with respect to $S$. The maximum of $f(x)$ at an element $A$ is defined to be the upper limit of the maxima at $A$ with respect to all possible sequences of elements different from $A$ whose only limit. ing element is $A$. The minimum at $A$ is similarly defined. The oscillation at $A$ is the difference between the maximum and the minimum. $\|$

Let $L$ be a limiting element of a sequence $A_{1}, A_{2}, \ldots, A_{n}, \ldots$ such that the oscillation of $f(x)$ at $A_{n}$, for all values of $n$, is not less than $k$. If the maxima $M_{n}$ of $f(x)$ at $A_{n}$ are not less than a fixed number $H$ for an infinite

* Hilbert, Wesen and Ziele einer Analysis der unendlichvielen unabhängigen Variabeln, Rendiconti del Circolo Matematico di Palermo, vol. 27 (1909). Also see HinberT's Mitteil_ ungen über die Grundzüge einer allgemeinen Theorie der linearen Integralgleichungen (vierte und fünfte) Göttinger Nachrichten, 1906.

† Hilbert, Palermo Rendiconti, loc. cit., p. 3.

†Fréchet, Nouvelles Annales de Mathématiques, 4th series, vol. 8, March and July, 1908. Strictly, by the wording of pages 1 and 23 ( July), it would appear that the Heine-Borel theorem does not hold for the Hilbert space ; this is bowever only due to a change of nomenclature, as a closer inspection enables the reader to prove.

§ Compare, for example, BAIRE, Leçons sur les tonctions discontinues, p. 73.

|| Compare BAIRE, loc. cit., p. 70 ; and a paper by the writer, Bulletin of the American Mathematical Society, vol. 13 (1907), p. 378. 
number of values of $n$, it follows that the maximum $M_{L}$ of $f(x)$ at $L$ is not less than $H$; for if $n_{1}, n_{2}, \cdots, n_{i}, \ldots$ are the values of $n$ for which $M_{n_{i}} \geqq H$, we can select a set of elements $A_{n_{i}}^{(1)}, A_{n_{i}}^{(2)}, \ldots, A_{n_{i}}^{(j)}, \ldots$ which approach $A_{n_{i}}$, and for which $f(x)$ exceeds $H-\epsilon$, where $\epsilon$ is an arbitrarily small fixed number independent of $i$. Since $L$ is, by section 1 , in the first derivative of $A_{n_{i}}^{(j)}$, the maximum at $L$ is not less than $H$.

Likewise if the minima $m_{n}$ of $f(x)$ at $A_{n}$ are not greater than $K$ for an infinite number of values of $n$; the minimum $m_{L}$ of $f(x)$ at $L$ is not greater than $K$. Suppose that the maximum $M_{L}$ and the minimum $m_{L}$ of $f(x)$ at $L$ differ by $k-4 \delta$. Then there are surely only a finite number of values of $n$ for which $M_{n}$ exceeds $M_{L}+\delta$ and only a finite number of values of $n$ for which $m_{n}$ is less than $m_{L}-\delta$; hence there are only a finite number of values of $n$ for which $M_{n}-m_{n}$ exceeds $k-2 \delta$, which is contrary to hypothesis. Therefore the oscillation, $M_{L}-m_{L}$, of $f(x)$ at $L$ is not less than $k$; and the theorem is proved. It results also from the proof just given that *

TheOrem 4. The maximum of $f(x)$ is itself a function which is semicontinuous above; and the minimum is semi-continuous below.

Another theorem which remains true is :

TheOREM 5. The frontier of any set is closed.

The frontier of a set $(E)$ of $(D)$ is defined to be the elements of $(E)$ which are limiting elements of elements not in $(E)$ together with the elements not in ( $E$ ) which are limiting elements of elements of $(E)$. Let $A$ be any limiting element of elements of the frontier. If $A$ is a limiting element of frontier elements $f_{1}, f_{2}, \cdots, f_{n}, \cdots$ which belong to $(E)$, each $f_{i}$ is a limiting element of elements not in $E$; hence $A$ is a limiting element of elements not in $E$. Hence $A$ is a limiting element of elements of $(E)$ and also of elements not in $(E)$; it is therefore a frontier element. A similar argument applies in case $A$ is a limiting element of frontier elements not in $(E)$.

That the several theorems proved above hold true without any restrictions on the definition of distance, or any connection between that definition and the definition of limiting element, is especially remarkable because the proofs of the analogous theorems for real numbers apparently make essential use of the distance notion. That such is the case, however, is demonstrated here by the entire absence of any definition of distance. $\dagger$

It is also clear that the property emphasized in section 1 - that the derivative of any set be closed - may be used to characterize a type of domains (with assigned definitions of limiting elements) which have in common some fundamental properties.

* Compare BaIre, loc. cit., p. 72.

† See Schornfliks, Bericht, loc. cit., p. 289. Compare, however, Vkblen, these Transactions, vol. 6 (1905), p. 167. 
Another important theorem which follows from these same assumptions occurs incidentally in the next section.

6. Enclosable Domains. In the theory of linear point sets the following statement may be taken as axiom: If the interval $\left(A_{i}, B_{i}\right)$ is interior to the interval $\left(A_{i-1}, B_{i-1}\right)$ and if the distance $\overline{A_{i} B_{i}}$ approaches zero, there is a single point common to all these intervals.

We shall now assume the analogous property : Corresponding to any element $A$ of $(D)$ there exists a countable set of closed assemblages $Q_{i}(A)$ which contain the single common interior element $A$, and which have the following properties :

(a) $Q_{i}(A)$ is interior to $Q_{i-1}(A)$.

(b) Corresponding to any integer $m$ an integer $n$ exists, such that, for any point $B$ whatever, any $Q_{n}$ which contains $B$ is interior to $Q_{m}(B)$.

When the domain $D$ with its definition of limiting element satisfies this requirement, we shall say that $D$ has the enclosable* property. All of the assemblages $Q$ are supposed to be selected now, and are held fixed in all that follows. Any $Q_{i}$ is said to be of rank $i$.

Several consequences of these assumptions may be stated at once:

Lemma 1. The fundamental domain $(D)$, if it contains more than a single element, is perfect. For any isolated point of $(D)$ is "interior" to any assemblage whatever. And $(D)$ is closed, by its definition.

Lemma 2. If $A_{i}$ is interior to $Q_{i}(B)$, for all values of $i, B$ is the only limiting element of the elements $A_{i}$. For since $B$ is the only element interior to all the $Q_{i}(B)$, any element $C$ distinct from $B$ may be enclosed in $Q_{j}(C)$, so that $Q_{j}(C)$ and $Q_{i}(B)$ have no elements in common for sufficiently high values of $i$ and $j$. Then the lemma follows from Theorem 1 .

It follows also from Theorem 1 , that a necessary and sufficient condition that $\operatorname{Lim}_{i=\infty} A_{i}=B$ is that at least one of the $A_{i}$ lies in $Q_{j}(B)$ for every value of $j$.

Lемма 3. If a sequence $A_{i}$ have the single limiting element $B$, corresponding to any fixed integer $m$, an integer $N$ exists such that $B$ is interior to $Q_{m}\left(A_{N+p}\right)$ for all values of $p$.

For a given $m$ select $n$ by $(b)$; since $Q_{n}(B)$ contains $A_{N+p}$, where $N$ is determined by Theorem $1, Q_{n}(B)$, and therefore $B$ itself, is interior to $Q_{m}\left(A_{N+p}\right)$, by $(b)$.

Lемма 4. If $B$ is interior to $(E)$, a number m exists such that any $Q_{m}$ which contains $B$ is interior to $(E)$.

\footnotetext{
* My attention is called to the similarity between this hypothesis and that given by VEBLEN for point sets, these Transactions, vol. 6 (1905), p. 168. The notion of uniformity of enclosure is essential in both cases. It is suggested that the word "enclosable" might read " uniformly enclosable" ; the former term is retained here, however, for simplicity.
} 
For let $R_{i}$ be a point of $Q_{i}(B)$ which is not interior to $(E)$. By Lemma 2, $\operatorname{Lim}_{i=\infty} R_{i}=B$, and all but a finite number of the $R_{i}$ are interior to $(E)$ by Theorem 1. It follows from (b) that $n \iota$ exists as stated in the theorem.

LemмA 5. If $B$ is interior to $(E)$ and if $\operatorname{Lim}_{i=\infty} A_{i}=B$, where $A_{i}$ is an element interior to a certain assemblage $Q_{i}^{\prime}$ of the type $Q_{i}$ for every value of $i$, then $n$ exists such that $Q_{n+p}^{\prime}$ is interior to $(E)$ for all values of $p$.

For $Q_{i}\left(A_{j}\right)$ contains $B$ and lies in $(E)$ for $i=m, j \geqq N$ by Lemma 4 and Lemma 3. Corresponding to $m$, a number $n$ exists, by (b), such that any $Q_{n}$ which contains $A_{j}$ is interior to $Q_{m}\left(A_{j}\right)$; hence $Q_{n+p}^{\prime}$ is interior to $Q_{m}\left(A_{n+p}\right)$ and therefore to $(E)$, if also $n \geqq N$.

Theorem 6. If any sequence $Q_{i}^{\prime}$ of assemblages of the types $Q_{i}$ $(i=1,2,3, \ldots, n, \ldots)$ are such that $Q_{i}^{\prime}$ is interior to $Q_{i-1}^{\prime}$, there is one and only one point $B$ interior to all $Q_{i}^{\prime}$, and also $\operatorname{Lim}_{i=\infty} A_{i}=B$ if $A_{i}$ is any element interior to $Q_{i}^{\prime}$.

For if $A_{i}$ is interior to $Q_{i}^{\prime}$ the sequence $A_{i}$ has at least one limiting element, $B$, and $B$ is interior to every $Q_{i}^{\prime}$. Any other element $C$ distinct from $B$ can be enclosed by $Q_{j}(C)$ so that $Q_{j}(C)$ and $Q_{i}(B)$ have no elements in common. But $Q_{i}(B)$ contains $Q_{k}^{\prime}$ for some value of $k$, by Lemma 5 ; hence $Q_{j}(C)$ contains at most a finite number of the points $A_{i}$. It follows that $B$ is the only element common to all the $Q_{i}^{\prime}$, and is the only limiting element of $A_{i}$.

Theonem 7. (Cauchy Fundamental Theorem.) The necessary and sufficient condition that the sequence $A_{j}$ have a single limiting element is that, corresponding to any arbitrarily assigned value of $i, a$ number $n$ exists such that, for all values of $p$, all the elements $A_{n+p}$ are interior to a single assemblage of the type $Q_{i}$.

The condition is necessary. For if $\operatorname{Lim}_{j=\infty} A_{j}=B$, a number $m$ exists by Lemma 3 , such that $Q_{i}\left(A_{m}\right)$ contains $B$; hence $Q_{i}\left(A_{m}\right)$ contains $A_{n+p}$ for sufficiently high values of $n$, by Theorem 1 .

The condition is sufficient. For if $Q_{i}$ contains $A_{n+p}$, the sequence $A_{n+p}$ has at least one limiting element $B$; if there were another such limiting element $C, Q_{k}(C)$ and $Q_{k}(B)$ would contain no common element for sufficiently high values of $k$; but $Q_{k}(B)$ contains $Q_{i}$, by $(b)$, for sufficiently high values of $i$, whence $Q_{k}(C)$ contains at most a finite number of the elements $A_{j}$, and is therefore not a limiting element.

Theorem 8. The set $P$ of elements of condensation* of any condensed $\dagger$ assemblage $E$ is either vacuous or perfect.

It can be shown that $P$ is closed without the assumption of the enclosable

\footnotetext{
* A limiting element is an element of condensation if it remains a limiting element after any countable assemblage is removed. See FrÉCHET, loc. cit., p. $6, \S 8$.

$\dagger \mathrm{A}$ condensed set is one for which any non-countable set of elements gives rise to at least one point of condensation. See FrÉch ET, loc. cit., p. 19, footnote.
} 
property. For let $A$ be a limiting element of elements of $P$, then $A$ belongs to $\left(E^{\prime}\right)$, since $P$ belongs to $\left(E^{\prime}\right)$, and $\left(E^{\prime}\right)$ is closed. If a countable set of elements are removed from $(E), P$ remains intact; hence $A$ remains a limit point of $P$, and $A$ remains a limit point of the remaining $(E)$. Hence $A$ belongs to $(P)$ * $^{*}$

Let us now show that $(P)$ is dense on itself; let $A$ be any point of $(P)$. Surround $A$ by a set of assemblages $\left(Q_{i}\right)$. Then there are an infinite number of values of $i, i_{1}, i_{2}, \ldots, i_{n}, \ldots$, for which a non-countable set of elements of $(E)$ are interior to $\left(Q_{i-1}\right)$, but not to $\left(Q_{i}\right)$, since otherwise a value of $i$ exists for which only a countable set of elements of $(E)$ are interior to $Q_{i}$, and this, by Theorem 1 , contradicts the hypothesis that $A$ belongs to $(P)$. Since $(E)$ is condensed, there is therefore at least one element of $(P)$ in $Q_{i_{n-1}}$, but not in $Q_{i_{n}+1}$, for all values of $i_{n}$. Let these be $p_{1}, p_{2}, \ldots, p_{n}, \ldots$ Then $A$ is the limit point of $p_{n}$, by Theorem 6 .

Theorem 9. The set of elements $(C)$ of any condensed assemblage $(E)$ which are not elements of condensation is countable.

For every element $k$ of $(C)$ is such that no element of condensation lies in $Q_{i}(k)$, where $i$ can be found when $k$ is given. The set of elements $\left(C_{i}\right)$ for which $i$ has the same fixed value is countable, for otherwise an element of condensation $p$ determined by $\left(C_{i}\right)$ would lie in $Q_{i}(k)$ for some element $k$ of $\left(C_{i}\right)$, by Lemma 3 . Hence the entire assemblage $(C)$ is countable.

It follows that every CLOSED condensed assemblage $(E)$ consists of a countable set and $a$ set which is either vacuous or perfect. The conclusion is still valid if $(E)$ is not closed but contains all its points of condensation.

Finally, any condensed assemblage $(E)$ consists of a countable set and a set of which every point is a point of condensation; the latter set is dense on itself.

With a modification of Fréchet's proof (Thesis, p. 20) similar to the modification of Lindelöf's proof $\dagger$ of Theorem 9 above, it can be shown that

Theorem 10. The elements of any assemblage $(E)$ which do not belong to its first derived assemblage $\left(E^{\prime}\right)$ form a countable set.

Hence, if any derived assemblage is countable, the original assemblage is countable.

Precisely following the proofs of Fréchet (Thesis, pages 20-22) we may now show that:

Theorem 11. Any closed assemblage $(E)$ is the first derivative of some assemblage selected from $(D)$.

Theorem 12. Any closed sub-assemblage $(F)$ of an assemblage $(E)$ can be formed from $(E)$ by omitting from $(E)$ the points interior to a countable number of the assemblages $Q$.

\footnotetext{
* See Fréchet, Palermo Rendiconti, vol. 30 (1910), p. 23, footnote. The closure property of section 1 above is the principle needed in FRÉCHET's thesis, p. 19, $\$ 29$.

† BokEL, Lesons, page 5.
} 
On account of its importance in what follows we shall repeat Fréchet's proof, which requires alteration in certain details, in the following special case:

If we select $\left(F^{\prime}\right)$ to be vacuous and $(E) \equiv(D)$, it results that

Theorem 13. Every point of $(D)$ is interior to at least one of a countable set of assemblages $Q . *$

Select some $Q$ at random, say $Q^{(0)}$. Then any point $A$ of $(D)$ not belonging to $Q^{(0)}$ has the property that $Q_{i}(A)$ has no point in common with $Q^{(0)}$ for sufficiently high values of $i$. We shall show that there exists a countable set of elements of $(D)$ :

$A_{1}^{(1)}, \ldots, A_{1}^{\left(p_{1}\right)} ; A_{2}^{(1)}, \ldots, A_{2}^{\left(p_{2}\right)} ; \ldots A_{n}^{(1)}, \ldots, A_{n}^{\left(p_{n}\right)} ; \ldots$ such that (a) $Q_{n}\left(A_{n}^{(j)}\right)$ has no point in common with $Q^{(0)}$, and $(b)$ any element of $(D)$ belongs to $Q^{(0)}$ or is interior to $Q_{n}\left(A_{n}^{(j)}\right)$ for some values of $n$ and $j$. For suppose the process carried out to any stage, so that the points up to $A_{n-1}^{\left(p_{n-1}\right)}$ are selected. Suppose $A$ exists such that $Q_{n}(A)$ contains no point in common with $Q^{(0)}$ and that $A$ is not interior to $Q_{k}\left(A_{k}^{(j)}\right)$ for $k<n$. Take this $A$ to be $A_{n}^{(1)}$; and select another $A$ of $(D)$ which has the same properties and which is not interior to $Q_{n}\left(A_{n}^{(1)}\right)$. Continuing in this way, form $A_{n}^{(1)}, A_{n}^{(2)}, \ldots$ This suite is finite, for if not the limit point $A_{n}^{(0)}$ would exist and $Q_{n}\left(A_{n}\right)$ would contain $A_{n}^{(0)}$ by Lemma 3 , and therefore also $A_{n}^{(m+p)}$, by Theorem 1 , for sufficiently high values of $m$.

This completes the proof if we note that every point of $Q^{(0)}$ is interior to that $Q$ which precedes $Q^{(0)}$ in the sequence of $Q$ 's which belong to the same point $A_{0}$ as does $Q^{(0)}$.

Since every $Q_{n}\left(A_{n}^{(j)}\right)$ may be replaced by $Q_{k}$, where $k<n$, since $Q_{n}\left(A_{n}^{(j)}\right)$ is interior to $Q_{k}\left(A_{n}^{(j)}\right)$, it follows that every point of $(D)$ is interior to at least one of a countable set of assemblages $Q_{k}$ of the same rank, $k$.

But, in the preceding proof, all of the points up to and including $A_{n-1}^{\left(p_{n}-1\right)}$ may be omitted; hence it follows that

TheORem 14. Every element of the fundamental domain $(D)$ is interior to a countable number of the assemblages $Q_{k}$, for any preassigned value of $k$.

Hence also, by Theorem 2, every element of $(D)$, or of any closed subset of $D$, is interior to a finite number of the assemblages $Q_{k}$, for any preassigned value of $k$.

Theorem 15. The domain $(D)$ itself is the first derived assemblage of a certain countable set of its own elements ; that is, $(D)$ is " separable." $\dagger$

For let us take the set of elements

$$
A_{0} ; A_{1}^{(1)}, \ldots, A_{1}^{\left(p_{1}\right)} ; A_{2}^{(1)}, \ldots, A_{2}^{\left(p_{2}\right)} ; \cdots
$$

defined in the proof of Theorem 13, and the elements

$$
A_{0_{k}} ; A_{(k+1)_{k}}^{(1)}, \cdots, A_{(k+1)_{k}}^{\left(p_{k+1}\right)} ; A_{(k+2)_{k}}^{(1)}, \cdots, A_{(k+2)_{k}}^{\left(p_{k+2}\right)} ; \cdots
$$

* See Fréchet, Thesis, p. 25.

† See Fréchet, Thesis, p. 23. 
obtained in the same proof by omitting the points $A_{1}^{(1)}, \cdots, A_{k}^{\left(p_{k}\right)}$ and by choosing $Q^{(0)}$ to be at least of rank $k+2$, as in the proof of Theorem 13. All of these points form a countable set; we shall show that any element of $(D)$ is a limiting element of this set.

For let $A$ be any element of $(D)$. By Theorem 13, $A$ is interior to $Q_{n}\left(A_{n}^{(j)}\right)$ for definite values of $n$ and $j$. With this fixed value of $n$, let us select $m$ by Lemma 4 so that any $Q_{m}$ which contains $A$ lies in $Q_{n}\left(A_{n}^{(j)}\right)$. Then choosing $k \geqq m$, select $r$ so that $A$ lies in $Q_{k+r}\left(A_{(k+r) k}^{(s)}\right)$. Proceeding in the same way, we may locate $A$ in the interior of a sequence of assemblages $Q^{(1)}$, $Q^{(2)}, \ldots$ such that $Q^{(n)}$ is of higher rank than $Q^{(n-1)}$ and interior to it, and such that $Q^{(n)}$ contains a point $A^{(n)}$ of the countable set selected above. It follows, by Theorem 6 , that $\operatorname{Lim}_{n=\infty} A^{(n)}=A$.

Corollary. Any subset of $(D)$ which is dense on itself is contained in the first derived assemblage of a countable set of its own elements.

TheOREM 16. Let $(E)$ be an assemblage, each of whose elements is interior to at least one of a family $(H)$ of assemblages $I$, where $(H)$ is not necessarily countable; then the necessary and sufficient condition that every point of $(E)$ is interior to at least one of a finite number of the assemblages $I$ is that $(E)$ be closed.

The condition is sufficient. For, since every element $e$ of $(E)$ is interior to some assemblage $I$, there is an upper limit to the value of $n$ for which $Q_{n}(e)$ is not interior to some $I$. If not, let $e_{1}, e_{2}, \ldots, e_{n}, \ldots$ be a set of elements of $(E)$ such that $Q_{n}\left(e_{n}\right)$ is not interior to any $I$. Let $e_{0}$ be one of the limiting elements of $e_{n}$; we may suppose without loss of generality that the $e_{n}$ have been already selected so that $\lim _{n=\infty} e_{n}=e_{0}$. Let $I_{0}$ be an assemblage of $(H)$ to which $e_{0}$ is interior. Then $Q_{n+p}\left(e_{n+p}\right)$ is interior to $I_{0}$ for sufficiently large values of $n$, by Lemma 5 .

Let $N$ be the upper limit on $n$ for which $Q_{n}(e)$ is not interior to some $I$. Select the finite set of assemblages $Q$ of $\operatorname{rank} N+1$ for which every point of $(D)$ is interior to some $Q_{N+1}$, by Theorem 14 and Theorem 2. Let $Q^{(1)}, Q^{(2)}$, $\ldots, Q^{(k)}$ be the subset of these $Q$ 's each of which contain at least one point of $(E)$. Select $I^{(1)}, I^{(2)}, \ldots, I^{(k)}$ such that $Q^{(i)}$ is interior to $I^{(i)}$; then every point of $(E)$ is interior to one of this finite set of $I$ 's. *

The condition is also necessary. For if $(E)$ is not closed, let $e_{1}, e_{2}, \ldots$, $e_{n}, \ldots$ be a set of elements of $e$ whose only limiting element $e_{0}$ does not belong to $(E)$; and consider the family $(H)$ of assemblages $I_{n}$ whose elements belong to $(E)$ but not to $Q_{n}\left(e_{n}\right)$. Not all the points $e_{i}$, and, $a$ fortiori, not all the points of $(E)$ can belong to a finite number of the $I_{n}$, by Theorem 7 .

\footnotetext{
* This proof follows in spirit that of FRÉCH ET, Thesis, p. 26, but in style it follows a proof by Bagnera, Rendiconti del Circolo Matematico di Palermo, vol. 28 (1909), p. 244, which is essentially the same as FRÉcHET's.
} 
Theorem 17. Every non-countable assemblage $(E)$ of $(D)$ is condensed.

Given a non-countable set of elements of $(E)$, at least one of the finite set of assemblages $Q_{k}$ of rank $k$, to at least one of which each point of $(E)$ belongs, contains a non-countable set of elements of $(E)$. Hence a sequence $Q_{1}^{(1)}, Q_{2}^{(2)}$, $Q_{3}^{(3)}, \ldots, Q_{n}^{(n)}$, of the type $Q$ exists, such that $Q_{n}^{(n)}$ is interior to $Q_{n-1}^{(n-1)}$ and that $Q_{n}^{(n)}$ contains a non-countable set of elements of $(E)$. Hence, by Theorem 6 , a point $A$ exists which is the only point interior to all of the $Q_{n}^{(n)}$ and which is a point of condensation of $(E)$.

From Theorems 9 and 17 we now conclude :

TheOREM 20. Every closed assemblage $(E)$ consists of a countable set together with a set which is perfect.

We have now proved a set of theorems analogous to the principal fundamental theorems of the theory of bounded sets of points; for these the only assumptions made were those of $\S 1$ and $\S 6$. The hypothesis that the first derived assemblage of every set is closed is therefore seen to be a fundamental hypothesis of farreaching character. The fundamental domains which possess that property include many important examples besides the original bounded sets of points, and have characteristics in common which make that class of domains one of great importance.

It should also be noticed that the enclosable property of section 6 is possessed by all ordinary domains under any of the usual definitions of distance, if $Q_{i}(A)$ be taken to be the spheroid about $A$ as center, with radius $1 / i$. 